\title{
Wiederaufnahme der Arbeit nach traumatischen Handverletzungen: medizinische, persönliche und arbeitsplatzbezogene Faktoren
}

\author{
Return to Work after Traumatic Hand Injuries: Medical, Personal and Work-related \\ Factors
}

Autoren

Institute

\author{
E. Oberfeld ${ }^{1}$, M. Zwahlen², E. Vögelin
}

${ }^{1}$ Klinik für Plastische- und Handchirurgie, Universitätsspital Bern, Inselspital, Bern, Schweiz ${ }^{2}$ Institut für Sozial- und Präventivmedizin, Universität Bern, Bern, Schweiz
Schlüsselwörter

- Handverletzung

- Prävention von Handverletzungen

- Rückkehr zur Arbeit

- Zeitdauer der Arbeitsunfähigkeit

\section{Key words}

- hand injury

- prevention of hand injury

- return to work

time off work eingereicht $\quad 6.10 .2014$

akzeptiert $\quad 27.12 .2014$

\section{Bibliografie}

Dol http://dx.doi.org/ 10.1055/s-0034-1398661

Online-Publikation: 10.2.2015

Handchir Mikrochir Plast Chir

2015; 47: 44-57

(c) Georg Thieme Verlag KG Stuttgart · New York

ISSN 0722-1819

Korrespondenzadresse Elisabeth Oberfeld lic. phil; MPH

Klinik für Plastische- und

Handchirurgie

Universitätsspital Bern,

Inselspital

Freiburgstraße

Bern

Switzerland 3010

elisabeth.oberfeld@insel.ch

\section{Zusammenfassung}

$\nabla$

Ziel: Die Studie untersucht anhand klinikbasierter Daten die Wiederaufnahme der Arbeit und die Dauer der Arbeitsunfähigkeit (AUF) von Patienten nach offenen Handverletzungen und wertet mögliche beeinflussende Faktoren quantitativ aus.

Methode: Eingeschlossen in die retrospektive Erhebung wurden 18-65 jährige Patienten mit akuten Handverletztungen $(n=435)$, die 2008 und 2009 in unserer Klinik operativ versorgt wurden. Durch versandte Fragebogen in 2011 wurden zu den Angaben aus der Krankengeschichte zusätzliche demografische, arbeitsbezogene und persönliche Informationen erhoben. In Gruppenvergleichen und multivariablen linearen Regressionen wurden Zusammenhänge zwischen möglichen beeinflussenden Faktoren und der Dauer der AUF ermittelt.

Ergebnisse: Die Stichprobe umfasste $290 \mathrm{~Pa}-$ tienten mit einem durchschnittlichen Alter von 38,9 (SD 13,2) Jahren. 98,6\% der Handverletzten nahmen nach einer Zeitdauer von 45,5 Tagen (Median) ihre Tätigkeit wieder auf. Klinische, soziodemografische und arbeitsbezogene Faktoren waren in den einfachen Gruppenvergleichen mit der Dauer der AUF assoziiert. Die Verletzungsregion, die Anzahl der betroffenen Regionen, das Aufkommen von Sekundäreingriffen, das Alter und die berufliche Tätigkeit waren die einzigen Variablen, die in der multivariablen linearen Regression einen statistisch signifikanten Zusammenhang mit der Dauer der AUF aufwiesen.

Schlussfolgerung: Verletzungsbezogene Faktoren und das Alter beeinflussen die Zeitdauer bis zur Wiederaufnahme der Arbeit nach einer Handverletzung maßgebend. Da sich beide nicht modifizieren lassen, kommt der Prävention von Handverletzungen, gefolgt von einer frühen sachgemäßen klinischen und therapeutischen Rehabilitation größte Bedeutung zu. Eine ebenso

\section{Abstract}

$\nabla$

Purpose: This study aimed to examine the work-related impact of open hand injuries, specifically, the amount of lost work days subsequent to the injury and factors associated with work-related rehabilitation.

Patients and Methods: We retrospectivley included consecutive patients with acute hand injuries who were operated between 2008 and 2009 in the Division of Hand Surgery $(n=435)$ at the Department of Orthopaedic, Plastic and Hand Surgery. Information was obtained from the medical records and via a self-reported questionnaire sent out in 2011. Patients younger than 18 or older than 65 years, as well as the unemployed were excluded from the study. Descriptive group analysis was used to establish statistical relationships between time off work (TOW) and possible influencing variables. Multiple linear regression was applied to analyse the impact of injury, personal and/or work-related factors on TOW.

Results: The sample included 290 patients with a mean age of 38.9 (SD 13.2) years of whom $98.6 \%$ returned to work after a median absence of 45.5 days. Univariate analysis demonstrated an association of length of absence from work with socio-demographic, clinical and workrelated factors. Multiple regression analysis indicated that the location of injury, the number of injured regions, the need for secondary surgery, age, and the type of occupation were independently associated with TOW.

Conclusion: Most factors associated with TOW after traumatic hand injuries could not be influenced. Possible interventions should probably target improved injury prevention, optimal clinical treatment and rehabilitation starting early after injury. Whether improvements in communication and enhancement of cooperation between the treatment teams, the workplace and 
wesentliche Rolle für die Zeitdauer bis zur Arbeitsaufnahme kommt den Anforderungen der jeweiligen beruflichen Tätigkeit zu. Durch eine verbesserte Kommunikation zwischen medizinischem Behandlungsteam, Arbeitgeber, Versicherer und dem Handverletzten ließen sich bereits zu einem frühen Zeitpunkt entscheidende Weichenstellungen in der Rehabilitation vornehmen. Ob diese eine graduelle und frühere Arbeitswiederaufnahme ermöglicht, bleibt Gegenstand weiterer Untersuchungen.

\section{Einleitung}

$\nabla$

Mindestens jede 3. Person, die im Notfall einer chirurgischen Klinik behandelt wird, weist eine Handverletzung auf (Schweiz pro Jahr ca. 150000 Handverletzungen) [1]. In der Schweiz gibt es keine Datenbank, die alle Bevölkerungsteile und alle Unfallbereiche in ausreichender Qualität beschreibt [2]. In der Statistik der Unfallversicherung werden bei den Versicherten die meisten Traumata für das Handgelenk, die Hand und die Finger ausgewiesen. In der Berufsunfallversicherung (BUV) sind dies 32,1\% (2014: 807940 Fälle), in der Nichtberufsunfallversicherung (NBUV) und der Unfallversicherung von Arbeitslosen (UVAL) 16,8\% der Fälle (2014: 79513 Fälle) [3]. Nicht erfasst werden in dieser Statistik Kinder, Schüler, Studierende, nicht erwerbstätige Hausfrauen und -männer, Selbstständigerwerbende und Pensionierte.

In der Europäischen Union werden mithilfe der European Injury Database (IDB) ${ }^{1}$ Datengrundlagen zu allen Arten von Verletzungen bereitgestellt. So machen Handverletzungen in der Europäischen Union (EU) $21 \%$ aller Verletzungen aus, das sind 8,8 Millionen Fälle in der EU27, mit einer geschätzten durchschnittlichen Inzidenzrate von $18 / 1000^{2}$ [4].

Handverletzungen resultieren oft in Funktionsstörungen der Hand, können zu langer Arbeitsunfähigkeit (AUF) [5,6], sozialen und ökonomischen Schwierigkeiten führen [7].

Das Ausmaß einer allfälligen Funktionseinschränkung wird durch Faktoren wie Art der Verletzung, Behandlungsmodalität, konstitutionelle Heilungsfaktoren (Alter, Gewebebeschaffenheit, vorbestehende Erkrankungen), Art der therapeutischen Nachbehandlung und Komplikationen bestimmt [8]. Neben den Charakteristika der Handverletzung beeinflussen persönliche, soziale und Umweltfaktoren Verlauf und Dauer der AUF. So spielen der Bildungsgrad [6,9], die ausgeübte Tätigkeit [6,9,10], das Alter $[9,11]$ und die soziale Unterstützung [9] bei der Wiedererlangung der Arbeitsfähigkeit eine Rolle.

Bisher wurde in der Schweiz lediglich eine Studie über sozioökonomische Gesichtspunkte von Handverletzungen durchgeführt, die auf Daten der Schweizerischen Unfallversicherungsanstalt (SUVA) ${ }^{3}$ beruht, wobei Selbstständigerwerbende nicht erfasst wurden. Denise Rüegg [12] untersuchte 2004 die Zeitdauer der AUF nach distalen Radiusfrakturen. Dabei zeigte sich, dass Behandlungsart, Alter, Nationalität und sozioökonomische Stellung die Dauer der AUF statistisch signifikant beeinflussen.

\footnotetext{
${ }^{1}$ The European Injury Database (IDB) is an injury surveillance system based on accident \& emergency department data from selected hospitals in various Member States. https://webgate.ec.europa.eu/idb/

${ }^{2}$ EU Injury Database gepoolte Daten 2006, 2007 n = 404'000

${ }^{3}$ Die Schweizerische Unfallversicherungsanstalt (Suva) ist die größte Trägerin der obligatorischen Unfallversicherung in der Schweiz und versichert rund 110000 Unternehmen bzw. 2 Millionen Berufstätige und Arbeitslose gegen Berufsunfälle, Berufskrankheiten und außerberufliche Unfälle.
}

the insurance carrier may support a staged and earlier return to work remains to be investigated.
Handverletzungen machen nicht nur einen beachtlichen Anteil aller behandelten Verletzungen aus, sondern stellen auch eine erhebliche wirtschaftliche Belastung dar. In der 2012 publizierten Studie aus den Niederlanden [13] wurde berechnet, dass Hand und Handgelenksverletzungen an erster Stelle aller Verletzungen stehen, was die Kostenfolge betrifft. Dies weniger der direkten Gesundheitskosten wegen, sondern vor allem aufgrund der hohen Produktivitätsausfallskosten.

Gemäß neusten Berechnungen der SUVA besteht heute bei einer 7-8 monatigen Arbeitsunfähigkeit noch eine 50\%ige Chance, wieder in den Beruf zurückzukehren [14].

Studien zur Bedeutung biopsychosozialer Faktoren in Bezug auf die Wiederaufnahme der Arbeit nach einer Handverletzung wurden bisher in der Schweiz nicht durchgeführt. Ziel unserer empirischen Studie war es bei einer größeren Patientenpopulation eines Tertiärzentrums die Zeitdauer der AUF und die Rückkehrrate an den Arbeitsplatz von Handverletzten zu ermitteln und die möglichen beeinflussenden Faktoren quantitativ auszuwerten. Es interessierte, welche medizinischen und nicht-klinischen Faktoren wie Alter, Geschlecht, Art der Arbeit, Herkunft, Schul/Berufsbildung und Formen sozialer Unterstützung Einfluss auf das Ergebnis (Zeitdauer der AUF bzw. der Rückkehr zur Arbeit) haben. Weiter wurde die Zufriedenheit mit der wiedererlangten Funktion der Hand aus Patientensicht erhoben.

\section{Methodik \\ $\nabla$}

Patienten und Vorgehen

Alle akuten, offenen Verletzungen der Hand und des Unterarms, die an unserer Klinik in der Zeit vom 1.01.2008-31.12.2009 versorgt wurden und eine komplette Nachsorge aufwiesen, wurden erfasst.

Eingeschlossen wurden Personen, die im Verletzungsjahr 18-65 jährig waren, Verletzungen an einer Hand/Finger und/oder den distalen zwei Drittel eines Unterarms erlitten hatten, zum Verletzungszeitpunkt erwerbstätig waren oder sich in Ausbildung befanden und über ausreichende Deutschkenntnisse verfügten. Ausgeschlossen wurden Patienten mit zusätzlich erlittenen Verletzungen, Verbrennungen und Bisswunden. Auf dem Flowchart $\odot$ Abb. 1 ist die Rekrutierung dargestellt.

Die Datenerhebung erfolgte in einem ersten Schritt anhand der Krankengeschichte.

Um Faktoren für die Wiederaufnahme der Arbeit auch aus Patientensicht $\mathrm{zu}$ erfassen, entwickelten wir einen Fragebogen, dessen Verständlichkeit mit 6 Handpatienten im Beisein der Projektleiterin getestet und angepasst wurde.

Um die Rücklaufquote des Fragebogens zu erhöhen, informierten wir die potenziellen Studienteilnehmenden vor dem Versand telefonisch über das Forschungsvorhaben. Erfolgte innerhalb von 2 Wochen keine Rücksendung, wurden sie erneut telefonisch 


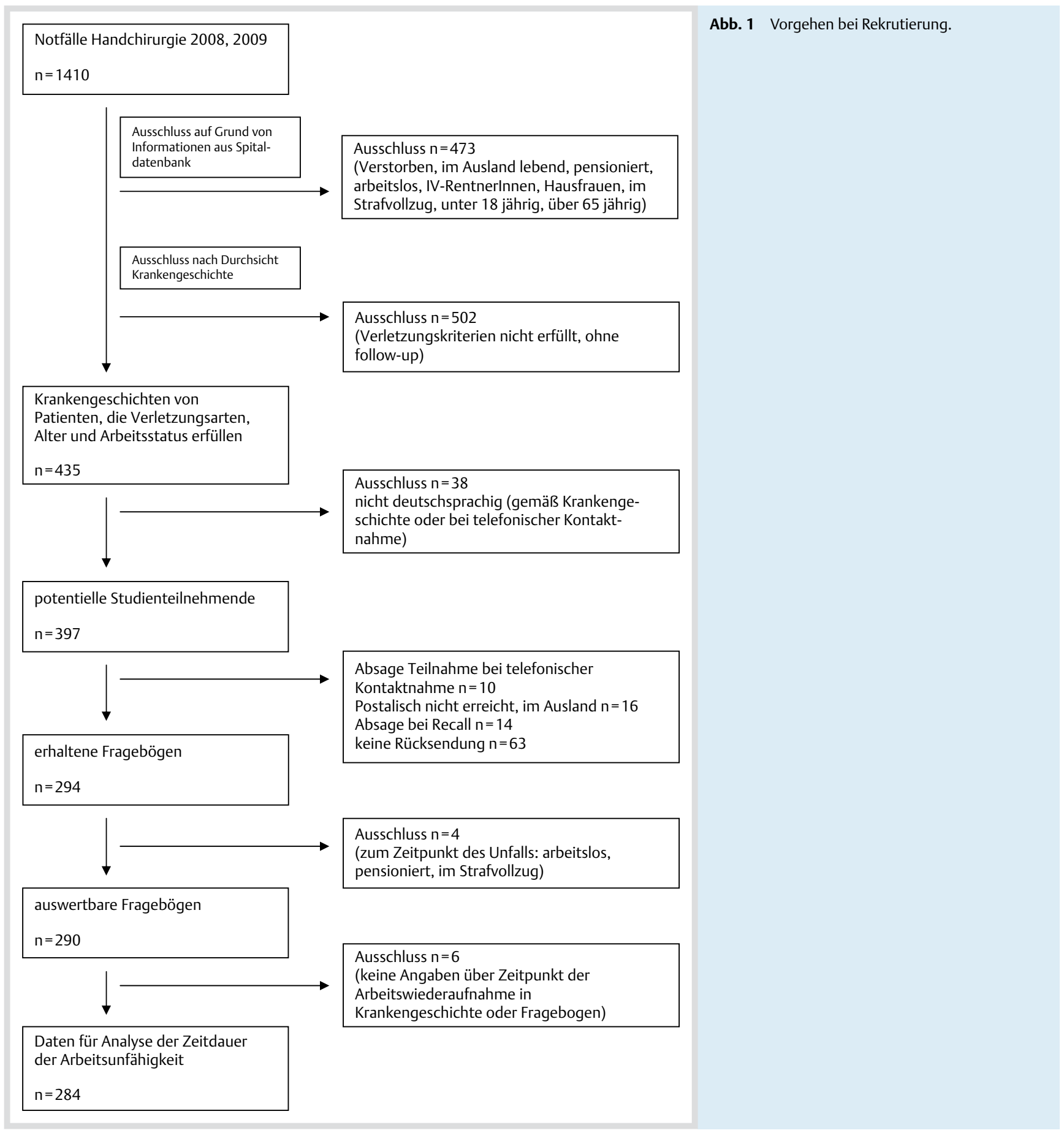

kontaktiert. Dem Fragebogen lagen Studieninformationen und eine Einverständniserklärung bei.

Alle Studienteilnehmenden gaben ihre schriftliche Einwilligung. Die Durchführung dieser Studie wurde von der Ethikkommission bewilligt.

\section{Berücksichtigte Faktoren und Instrumente}

Mit Bezug zum biopsychosozialen Rahmenmodell und nach Sichtung der relevanten Literatur wurden verletzungs- und tätigkeitsbezogene Faktoren, Unterstützungsfaktoren und Selbsteinschätzung mittels QuickDASH berücksichtigt (siehe online: e-Text Erläuterungen).

\section{Statistische Analyse}

Die Daten wurden zur statistischen Auswertung verschlüsselt, unter Zuhilfenahme der Software-Programms Epidata 3.2 (www.epidata.dk) elektronisch erfasst und mit der Statistiksoftware STATA 11.2 (StataCorp LP, College Station, Texas, USA) ausgewertet. Die Poweranalyse zeigte, dass ein Total von 160-200 Beobachtungen bei einer Standardabweichung von 1 (auf dem 10er- Logarithmus der Dauer) über $80 \%$ Power hat, um einen statistisch signifikanten mittleren Unterschied von 0,5 zu beschreiben.

Gruppenvergleiche wurden mittels $\mathrm{X}^{2}$-Test für kategorische Daten und mittels Wilcoxon-Mann-Whitney-Rangsummen-Test 
oder Kruskal-Wallis-Test für kontinuierliche Daten ausgeführt. Als Signifikanzniveau galt $\alpha=0,05,2$-seitig.

Die Hauptanalyse bezog sich auf den Zeitraum (in Tagen) von der ersten Operation nach der Handverletzung bis zur ersten Wiederaufnahme der Arbeit unabhängig vom Pensum oder allfälligen beruflichen Maßnahmen. Die Zeitvariable beruhte auf Angaben der Studienteilnehmenden, fehlende Angaben ergänzten wir mit Daten aus der Krankengeschichte.

Als mögliche Einflussvariablen wurden in einem ersten Schritt die vorgängig beschriebenen Faktoren einzeln in Bezug auf die Zeitdauer der AUF mittels $X^{2}$-Test untersucht. Variablen über die Zufriedenheit mit der Handfunktion und die soziale Unterstützung wurden für die Analyse dichotomisiert.

In einem zweiten Auswertungsschritt wurde der 10er-Logarithmus (aufgrund der nicht normalverteilten Daten) der Dauer mittels multivariabler linearer Regressionsmodelle analysiert, um festzustellen, welche Faktoren die Varianz in der Dauer der AUF erklären. Um Zusammenhänge zwischen der abhängigen Variablen und einzelnen beeinflussenden Variablen zu ermitteln, analysierten wir die Daten aufgrund empirischer Überlegungen in 4 Blöcken: relevante Verletzungsmerkmale, persönlichen Faktoren, arbeitsplatzbezogene Faktoren und Unterstützungsfaktoren. Die 4 Modelle wurden untereinander mittels LikelihoodRatio-Test verglichen. Die Resultate dieser Modelle werden als prozentuale Veränderung mit $95 \%$ Vertrauensintervall der Dauer der Arbeitsunfähigkeit dargestellt.

Fehlende Daten sind in den Gruppenvergleichen und in der Regressionsanalyse ausgewiesen.

\section{Ergebnisse}

\section{Allgemeine Beschreibung der Stichprobe}

Für die Auswertung des Fragebogens standen Daten von 290 Patienten zu Verfügung, die Rücklaufquote betrug $76 \%$. Die Gruppe der Antwortenden und die der Nicht-Antwortenden unterschieden sich in Bezug auf Alter und Nationalität, aber nicht bezüglich der Verletzungsmerkmale (siehe online: e-Tab. 1).

Die durchschnittliche Zeitdauer zwischen dem erlittenen Trauma und der Beantwortung des Fragebogens betrug 24 Monate (range: 14-37). Die Studienpopulation umfasste 240 (82,8\%) Männer und 50 (17,3\%) Frauen, das durchschnittliche Alter zum Operationszeitpunkt betrug 38,9 Jahre (SD 13,2).

Die Charakteristika der Studienteilnehmenden, bei welchen die Zeitdauer der AUF ermittelt werden konnte, sind in 0 Tab. 1 ersichtlich.

\section{Zeitdauer der Arbeitsunfähigkeit}

In der vorliegenden Untersuchung liegen Daten von 284 Studienteilnehmenden vor.

Die Zeitdauer der AUF konnte von 6 (2,0\%) Patienten weder aus dem Fragebogen noch aus der Krankengeschichte erhoben werden, wovon 2 Personen die Arbeit nicht wieder aufgenommen haben. Die Teilnehmenden mit und ohne Angabe der Dauer der AUF unterschieden sich nicht hinsichtlich demografischer und verletzungsbedingter Merkmale. $\mathbf{O}$ Abb. $\mathbf{2}$ zeigt die prozentualen Anteile der Arbeitswiederaufnahme anhand der Dauer der AUF. Innerhalb von 6 Wochen nahmen 46,1\% ihre Tätigkeit wieder auf, innerhalb von 12 Wochen waren es $72,5 \%$. Die durchschnittliche Dauer der AUF betrug 68 Tage (SD 96), der Median 45,5 Tage (range 0-901).
In $\odot$ Tab. 1 sind die Ergebnisse der beeinflussenden Variablen für die Zeitdauer bis zur Wiederaufnahme der Arbeit wiedergegeben. Die deskriptive Statistik zeigt Anzahl, Prozent, Mittelwert, 25ste und 75 ste Perzentile für sämtliche Charakteristika von 284 Studienteilnehmenden.

Frauen ( $\mathrm{p}: 0,017)$ und Personen in der Altersgruppe 54-65 (p:0,048) wiesen kürzere Arbeitsabwesenheiten auf. Je höher das Ausbildungsniveau, desto früher erfolgte der Arbeitsbeginn $(p:<0,001)$. Bezogen auf die verschiedenen Versicherer zeigten sich bei der Dauer der AUF signifikante Unterschiede (p: 0,030), allerdings gilt es zu berücksichtigen, dass der Anteil der manuell Arbeitenden bei der SUVA wesentlich höher war (p: $<0,001)$.

Verletzungsrelevante Faktoren, wie Art der verletzten Strukturen (p: 0,003), Anzahl betroffener Regionen (p: 0,002) und Lokalisation der Verletzung (p:0,009) präsentierten signifikante Werte. Verletzungen mehrer Regionen, die Beteiligung von Nerven und proximale Verletzungen verzögerten den Arbeitsbeginn, ebenso wie Komplikationen im Heilungsverlauf und Sekundäreingriffe ( $\mathrm{p}:<0,001)$.

Unfälle, welche am Arbeitsort erfolgten ( $p: 0,002)$, sowie vorwiegend manuelle berufliche Tätigkeiten $(p:<0,001)$ waren mit längeren Zeiten der AUF assoziiert. Ob jemand angestellt, selbstständig erwerbend war oder sich in Ausbildung befand, erwies sich für die Dauer der AUF als nicht signifikant, wie auch die berufliche Anforderung an die Handfunktion keine Relevanz erkennen ließ.

Unterstützungsfaktoren zeigten weder im privaten noch im Arbeitsbereich signifikante Zusammenhänge bezüglich der Dauer der AUF. Unterschiede gab es bei der Unterstützung durch die Versicherer (p:0,043). Personen, die früher ihre Arbeit aufnahmen, kannten keine Ansprechperson. Von den 78 Handverletzten, die länger als 12 Wochen arbeitsunfähig waren, verfügten $63(80,8 \%)$ seitens der Versicherer über keine Ansprechperson, nach einem halben Jahr waren es 9 von 19 Personen (Daten nicht abgebildet).

\section{Rückkehr an den früheren Arbeitsplatz}

266 (91,7\%) der Befragten gaben an, am bisherigen Arbeitsplatz ihre Tätigkeit wieder aufgenommen zu haben. Bei 6 Arbeitsplätzen wurden Anpassungen vorgenommen. Von den 24 Handverletzten, die nicht wieder an ihren früheren Arbeitsplatz zurückkehrten, nahmen 6 bei ihren bisherigen Arbeitgebern eine andere Tätigkeit an, 10 wechselten den Arbeitgeber, 1 machte sich selbstständig und 3 absolvierten eine berufliche Umschulung. 2 Studienteilnehmende haben seit dem Unfall ihre Arbeit nicht wieder aufgenommen und 2 machten keine Angaben.

Von den 286, die ihre Tätigkeit wieder aufnahmen, gaben $78 \%$ an, zu ihrem früheren Anstellungsgrad begonnen zu haben. 43 $(15,0 \%)$ Personen starteten mit einem Teilpensum (zeitliche Reduktion), 15 (5,2\%) mit einer Teilbelastung. Von diesen 58 gaben 41 an, zum Befragungszeitpunkt wieder vollumfänglich erwerbstätig zu sein.

Zum Befragungszeitpunkt gingen 82\% der Handverletzten wieder ihrer vorherigen Tätigkeit nach. Eine Person erhielt aus nicht die Handverletzung betreffenden Gründen eine volle Invalidenrente, 5 waren pensioniert und 9 gaben an, arbeitslos zu sein. 4 befanden sich in einer Ausbildung/Weiterbildung, 3 in der beruflichen Umschulung, 4 wechselten von einer vorwiegend manuellen zu einer Tätigkeit im Dienstleistungsbereich, 2 machten keine Angaben. 
Tab. 1 Charakteristika anhand Fragebogen + Krankengeschichte in Bezug auf die Dauer der Arbeitsunfähigkeit (AUF).

\begin{tabular}{|c|c|c|c|c|c|c|c|}
\hline & \multicolumn{4}{|c|}{ Dauer der AUF in Tagen $\mathbf{N}(\%)$} & \multicolumn{3}{|c|}{ Dauer der AUF in Tagen } \\
\hline & $\begin{array}{l}<14 \text { Tage } \\
\text { (< } 2 \text { Wo })\end{array}$ & $\begin{array}{l}\text { 15-27 Tage } \\
\text { (2-4 Wo) }\end{array}$ & $\begin{array}{l}\text { 28-83 Tage } \\
\text { (5-12 Wo) }\end{array}$ & $\begin{array}{l}>83 \text { Tage } \\
\text { (> } 12 \text { Wo })\end{array}$ & p-Wert & $\begin{array}{l}\text { Median } \\
\text { (p25-p75) }\end{array}$ & $\begin{array}{l}\text { Total } \\
\text { N (\%) }\end{array}$ \\
\hline Gesamt & $43(15,1)$ & $58(20,4)$ & $105(37,0)$ & $78(27,4)$ & $<0,001$ & $45,5(21-86)$ & $284(100,0)$ \\
\hline Geschlecht & & & & & 0,017 & & \\
\hline Männer & $36(15,3)$ & $40(17,0)$ & $90(38,3)$ & $69(29,4)$ & & $55(21-89)$ & $235(82,8)$ \\
\hline Frauen & $7(14,3)$ & $18(36,7)$ & $15(30,6)$ & $9(18,4)$ & & $25(16-48)$ & $49(17,3)$ \\
\hline Altergruppen & & & & & 0,048 & & \\
\hline$<25$ & $4(7,0)$ & $17(29,8)$ & $21(36,8)$ & $15(26,3)$ & & $42(21-84)$ & $57(20,1)$ \\
\hline $25-34$ & $8(14,0)$ & $12(21,1)$ & $24(42,1)$ & $13(22,8)$ & & $39(21-76)$ & $57(20,1)$ \\
\hline $35-44$ & $6(9,4)$ & $15(23,4)$ & $27(42,2)$ & $16(25,0)$ & & $50,5(21-84,5)$ & $64(22,5)$ \\
\hline $45-54$ & $11(19,0)$ & $7(12,1)$ & $18(31,0)$ & $22(37,9)$ & & $67(21-100)$ & $58(20,4)$ \\
\hline $55-65$ & $14(29,2)$ & $7(14,6)$ & $15(31,3)$ & $12(25,0)$ & & $43(9-81)$ & $48(16,9)$ \\
\hline Zivilstand Befragungszeitpunkt & & & & & 0,065 & & \\
\hline ledig & $12(10,0)$ & $34(28,3)$ & $44(36,7)$ & $30(25,0)$ & & $41(21-83,5)$ & $120(42,3)$ \\
\hline verheiratet & $27(20,2)$ & $19(14,2)$ & $48(35,8)$ & $40(29,9)$ & & $52(16-90)$ & $134(47,2)$ \\
\hline geschieden/verwitwet/getrennt & $4(13,8)$ & $5(17,2)$ & $13(44,8)$ & $7(24,1)$ & & $52(23-76)$ & $29(10,2)$ \\
\hline fehlende Angaben & $0(0,0)$ & $0(0,0)$ & $0(0,0)$ & $1(100,0)$ & & $100(100-100)$ & $1(0,4)$ \\
\hline Herkunft & & & & & 0,066 & & \\
\hline Schweiz & $36(15,7)$ & $47(20,4)$ & $90(39,1)$ & $57(24,8)$ & & $44(20-83)$ & $230(81,0)$ \\
\hline Nord-, West-, Osteuropa & $6(31,6)$ & $6(31,6)$ & $2(10,5)$ & $5(26,3)$ & & $21(7-84)$ & $19(6,7)$ \\
\hline Südeuropa, Türkei, Westbalkan & $0(0,0)$ & $3(13,6)$ & $9(40,9)$ & $10(45,6)$ & & $82(32-109)$ & $22(7,6)$ \\
\hline übrige Länder & $1(12,5)$ & $1(12,5)$ & $3(37,5)$ & $3(37,5)$ & & $70,5(37,5-139,5)$ & $8(2,8)$ \\
\hline fehlende Angaben & $0(0,0)$ & $1(20,0)$ & $1(20,0)$ & $3(60,0)$ & & $84(29-91)$ & $5(1,8)$ \\
\hline Aufenthaltsstatus in der Schweiz & & & & & 0,306 & & \\
\hline Schweizer Bürgerrecht & $38(15,5)$ & $49(20,0)$ & $95(38,8)$ & $63(25,7)$ & & $46(20-84)$ & $245(86,3)$ \\
\hline Niederlassung (Ausweis C) & $3(14,3)$ & $3(14,3)$ & $6(28,6)$ & $9(42,7)$ & & $60(23-109)$ & $21(7,4)$ \\
\hline Jahresaufenthalt (Ausweis B) & $1(6,7)$ & $5(33,3)$ & $4(26,7)$ & $5(33,3)$ & & $35(21-92)$ & $15(5,3)$ \\
\hline vorläufig aufgenommen (Ausweis F) & $0(0,0)$ & $0(0,0)$ & $0(0,0)$ & $1(100,0)$ & & $91(91-91)$ & $1(0,4)$ \\
\hline anderer Status & $1(100,0)$ & $0(0,0)$ & $0(0,0)$ & $0(0,0)$ & & $1(1-1)$ & $1(0,4)$ \\
\hline fehlende Angaben & $0(0,0)$ & $1(100,0)$ & $0(0,0)$ & $0(0,0)$ & & $25(25-25)$ & $1(0,4)$ \\
\hline höchste abgeschlossene Ausbildung & & & & & $<0,001$ & & \\
\hline ohne nachobligatorische Schulbildung & $1(3,03)$ & $7(21,2)$ & $13(39,4)$ & $12(36,7)$ & & $61(31-96)$ & $33(11,6)$ \\
\hline Sekundarstufe II (Berufsbildung) & $18(10,8)$ & $36(21,6)$ & $69(41,3)$ & $44(26,4)$ & & $52(21-85)$ & $167(58,8)$ \\
\hline Sekundarstufe II (Matura) & $3(21,4)$ & $3(21,4)$ & $5(35,7)$ & $3(21,4)$ & & $36(21-65)$ & $14(4,9)$ \\
\hline $\begin{array}{l}\text { höhere Berufsbildung (Meisterdiplom, } \\
\text { Fachschule) }\end{array}$ & $6(15,0)$ & $8(20,0)$ & $12(30,0)$ & $14(35,0)$ & & $46(19-90)$ & $40(14,1)$ \\
\hline Hochschule & $15(50,0)$ & $4(13,3)$ & $6(20,0)$ & $5(16,7)$ & & $12(3-42)$ & $30(10,6)$ \\
\hline Hauptverdiener & & & & & 0,106 & & \\
\hline nein & $4(8,9)$ & $15(33,3)$ & $13(28,9)$ & $13(28,9)$ & & $39(20-86)$ & $45(15,9)$ \\
\hline ja & $29(16,7)$ & $28(16,1)$ & $71(40,8)$ & $46(26,4)$ & & $55(21-86)$ & $174(61,3)$ \\
\hline Hälfte des Familieneinkommens & $9(20,0)$ & $11(24,4)$ & $12(26,7)$ & $13(28,9)$ & & $32(19-88)$ & $45(15,9)$ \\
\hline fehlende Angaben & $1(5,0)$ & $4(20,0)$ & $9(45,0)$ & $6(30,0)$ & & $42(27,5-87)$ & $20(7,0)$ \\
\hline Raucherstatus & & & & & 0,154 & & \\
\hline Raucher & $7(7,3)$ & $21(21,9)$ & $41(42,7)$ & $27(28,1)$ & & $55,5(24-87,5)$ & $96(33,8)$ \\
\hline ehemalige Raucher & $15(18,5)$ & $16(19,8)$ & $30(37,0)$ & $20(24,7)$ & & $42(21-83)$ & $81(28,5)$ \\
\hline Nichtraucher & $21(20,8)$ & $16(15,8)$ & $33(32,7)$ & $31(30,7)$ & & $52(17-88)$ & $101(35,6)$ \\
\hline fehlende Angaben & $0(0,0)$ & $5(83,3)$ & $1(16,7)$ & $0(0,0)$ & & $22,5(16-26)$ & $6(2,1)$ \\
\hline Versicherer & & & & & 0,030 & & \\
\hline SUVA & $16(10,7)$ & $24(16,0)$ & $63(42,0)$ & $47(31,3)$ & & $58,5(26-91)$ & $150(52,8)$ \\
\hline Unfallversicherung & $8(17,8)$ & $15(33,3)$ & $12(26,7)$ & $10(22,2)$ & & $27(14-66)$ & $45(15,9)$ \\
\hline Krankenkasse & $19(21,4)$ & $19(21,4)$ & $30(33,7)$ & $21(26,9)$ & & $38(14-75)$ & $88(31,3)$ \\
\hline Handdominanz & & & & & 0,948 & & \\
\hline adominante Hand & $23(16,4)$ & $29(20,7)$ & $49(35,0)$ & $39(27,8)$ & & $43(16-87)$ & $140(49,3)$ \\
\hline dominante Hand & $18(13,5)$ & $27(20,3)$ & $53(39,9)$ & $35(26,3)$ & & $52(21-84)$ & $133(46,8)$ \\
\hline Beidhändigkeit & $1(20,0)$ & $1(20,0)$ & $1(20,0)$ & $2(40,0)$ & & $35(24-103)$ & $5(1,8)$ \\
\hline fehlende Angaben & $1(16,7)$ & $1(16,7)$ & $2(33,3)$ & $2(33,3)$ & & $39,5(21-132)$ & $6(2,1)$ \\
\hline Verletzungstyp & & & & & 0,258 & & \\
\hline scharfe Schnittwunde ohne Defekt & $24(19,1)$ & $30(23,8)$ & $46(36,5)$ & $26(20,6)$ & & $31,5(14-69)$ & $126(44,4)$ \\
\hline scharfe Schnittwunde mit Defekt & $4(6,3)$ & $12(18,8)$ & $22(34,4)$ & $26(40,6)$ & & $61(31-103,5)$ & $64(22,5)$ \\
\hline Quetschwunde ohne Defekt & $8(15,4)$ & $10(19,2)$ & $19(36,5)$ & $15(28,9)$ & & $54(21-87,5)$ & $52(18,3)$ \\
\hline Quetschwunde mit Defekt & $5(15,6)$ & $6(18,8)$ & $13(40,6)$ & $8(25,0)$ & & $59(16-87,5)$ & $32(11,3)$ \\
\hline andere & $2(20,0)$ & $0(0,0)$ & $5(50,0)$ & $3(30,0)$ & & $61,5(30-92)$ & $10(3,5)$ \\
\hline
\end{tabular}


Tab. 1 Fortsetzung.

\begin{tabular}{|c|c|c|c|c|c|c|c|}
\hline & \multicolumn{4}{|c|}{ Dauer der AUF in Tagen $\mathbf{N}(\%)$} & \multicolumn{3}{|c|}{ Dauer der AUF in Tagen } \\
\hline & $\begin{array}{l}<14 \text { Tage } \\
(<2 \mathrm{Wo})\end{array}$ & $\begin{array}{l}\text { 15-27 Tage } \\
\text { (2-4 Wo) }\end{array}$ & $\begin{array}{l}\text { 28-83 Tage } \\
\text { (5-12 Wo) }\end{array}$ & $\begin{array}{l}>83 \text { Tage } \\
(>12 \mathrm{Wo})\end{array}$ & p-Wert & $\begin{array}{l}\text { Median } \\
\text { (p25-p75) }\end{array}$ & $\begin{array}{l}\text { Total } \\
\text { N (\%) }\end{array}$ \\
\hline Verletzte Struktur & & & & & 0,003 & & \\
\hline nur Haut & $8(32,0)$ & $10(40,0)$ & $6(24,0)$ & $1(4,0)$ & & $20(10-28)$ & $25(8,8)$ \\
\hline isoliert (K, BS, SS, Ge, M, G) & $6(16,2)$ & $8(21,6)$ & $16(43,2)$ & $7(18,9)$ & & $41(21-66)$ & $37(13,0)$ \\
\hline kombiniert (K, BS, SS, Ge, M, G) & $18(16,5)$ & $21(19,3)$ & $29(35,8)$ & $31(28,4)$ & & $53(18-86)$ & $109(38,4)$ \\
\hline Nerv & $8(15,4)$ & $9(17,3)$ & $23(44,3)$ & $12(23,1)$ & & $35(21,5-81,5)$ & $52(18,3)$ \\
\hline kombiniert (K, BS, SS, Ge, M, G)+ Nerv & $3(4,9)$ & $10(16,4)$ & $21(34,4)$ & $27(44,3)$ & & $75(36-146)$ & $61(21,5)$ \\
\hline Anzahl verletzte Regionen & & & & & 0,002 & & \\
\hline eine Region & $37(17,7)$ & $47(22,5)$ & $80(38,3)$ & $45(21,5)$ & & $38(16-76)$ & $209(73,6)$ \\
\hline 2 Regionen & $5(10,9)$ & $7(15,2)$ & $18(39,1)$ & $16(34,8)$ & & $60(27-96)$ & $46(16,2)$ \\
\hline 3 und mehr Regionen & $1(3,5)$ & $4(13,8)$ & $7(24,1)$ & $17(58,6)$ & & $105(65-194)$ & $29(10,2)$ \\
\hline Verletzte Region: & & & & & 0,009 & & \\
\hline Endglied & $16(23,9)$ & $17(25,4)$ & $22(32,8)$ & $12(17,9)$ & & $28(14-66)$ & $67(23,6)$ \\
\hline Finger & $20(12,8)$ & $35(22,4)$ & $57(36,5)$ & $44(28,2)$ & & $47,5(21-88)$ & $156(54,9)$ \\
\hline Mittelhand & $4(12,1)$ & $5(15,2)$ & $17(51,5)$ & $7(21,2)$ & & $44(21-67)$ & $33(11,6)$ \\
\hline Handgelenk+ distale 2/3 Unterarm & $3(10,7)$ & $1(3,6)$ & $9(32,1)$ & $15(53,6)$ & & $86,5(42-132)$ & $28(9,9)$ \\
\hline Komplikationen & & & & & $<0,001$ & & \\
\hline nein & $40(17,9)$ & $53(23,7)$ & $82(36,6)$ & $49(21,9)$ & & $35(17,5-73)$ & $224(78,9)$ \\
\hline ja & $3(5,0)$ & $5(8,3)$ & $23(38,3)$ & $29(48,3)$ & & $82,5(49,5-114)$ & $60(21,1)$ \\
\hline Sekundäreingriffe & & & & & $<0,001$ & & \\
\hline keine & $41(17,2)$ & $56(23,5)$ & $89(37,4)$ & $52(21,9)$ & & $38(18-75)$ & $238(83,8)$ \\
\hline einer & $2(5,6)$ & $2(5,6)$ & $14(38,9)$ & $18(50,0)$ & & $87(49-135,5)$ & $36(12,7)$ \\
\hline mehrere & $0(0,0)$ & $0(0,0)$ & $2(20,0)$ & $8(80,0)$ & & $199(92-285)$ & $10(3,5)$ \\
\hline Verletzungsort & & & & & 0,002 & & \\
\hline Arbeitsplatz & $16(9,2)$ & $35(20,1)$ & $74(42,5)$ & $49(28,1)$ & & $61,5(49,4)$ & $174(61,3)$ \\
\hline anderer & $27(24,6)$ & $23(20,9)$ & $31(28,2)$ & $29(26,4)$ & & $78,3(141,0)$ & $110(38,7)$ \\
\hline Arbeitsstatus zum Zeitpunkt des Unfalls & & & & & 0,293 & & \\
\hline angestellt & $31(14,4)$ & $46(21,3)$ & $80(37,0)$ & $59(27,3)$ & & $44,5(21-86)$ & $216(76,1)$ \\
\hline selbstständig & $9(20,0)$ & $8(8,9)$ & $19(42,2)$ & $13(28,9)$ & & $60(21-88)$ & $45(15,9)$ \\
\hline in Ausbildung & $3(13,0)$ & $8(34,8)$ & $6(26,1)$ & $6(26,1)$ & & $28(20-88)$ & $23(8,1)$ \\
\hline Beruf zum Unfallzeitpunkt & & & & & $<0,001$ & & \\
\hline manuell Tätige (blue collar) & $18(8,2)$ & $43(19,6)$ & $87(39,7)$ & $71(32,4)$ & & $59(25-91)$ & $219(77,1)$ \\
\hline andere (white collar) & $25(40,3)$ & $14(22,6)$ & $17(27,4)$ & $6(9,7)$ & & $20(7-38)$ & $62(21,8)$ \\
\hline fehlende Angaben & $0(0,0)$ & $1(33,3)$ & $1(33,3)$ & $1(33,3)$ & & $46(17-91)$ & $3(1,1)$ \\
\hline $\begin{array}{l}\text { Beruf derselbe zurzeit des Unfalls und } \\
\text { der Befragung: }\end{array}$ & & & & & 0,735 & & \\
\hline nein & $5(10,2)$ & $11(22,5)$ & $17(34,7)$ & $16(32,7)$ & & $44(23-91)$ & $49(17,3)$ \\
\hline ja & $38(16,3)$ & $46(19,7)$ & $87(37,3)$ & $62(26,6)$ & & $46(20-85)$ & $233(82,1)$ \\
\hline fehlende Angaben & $0(0,0)$ & $1(50,0)$ & $1(50,0)$ & $0(0,0)$ & & $33,5(21-46)$ & $2(0,7)$ \\
\hline $\begin{array}{l}\text { berufliche Anforderung an die Hand- } \\
\text { funktion }\end{array}$ & & & & & 0,184 & & \\
\hline minimal & $2(15,4)$ & $3(23,1)$ & $1(7,7)$ & $7(53,9)$ & & $90(22-106)$ & $13(4,6)$ \\
\hline mäßig & $12(19,1)$ & $9(14,3)$ & $26(41,3)$ & $16(25,4)$ & & $48(21-84)$ & $63(22,2)$ \\
\hline intensiv & $29(14,1)$ & $44(21,4)$ & $78(37,9)$ & $55(26,7)$ & & $43(21-86)$ & $206(72,5)$ \\
\hline fehlende Angaben & $0(0,0)$ & $2(100,0)$ & $0(0,0)$ & $0(0,0)$ & & $20(17-23)$ & $2(0,7)$ \\
\hline Wiederaufnahme der Arbeit: & & & & & 0,245 & & \\
\hline gleicher Arbeitsplatz & $40(15,6)$ & $54(21,0)$ & $97(37,7)$ & $66(25,7)$ & & $44(21-84)$ & $257(90,5)$ \\
\hline nicht derselbe Arbeitsplatz & $2(8,0)$ & $4(16,0)$ & $8(32,0)$ & $11(44,0)$ & & $81(32-122)$ & $25(8,8)$ \\
\hline fehlende Angaben & $1(50,0)$ & $0(0,00)$ & $0(0,0)$ & $1(50,0)$ & & $46,5(7-86)$ & $2(0,7)$ \\
\hline Anstellungsgrad nach der Verletzung & & & & & 0,030 & & \\
\hline gleich wie vorher & $38(17,3)$ & $51(23,2)$ & $78(35,4)$ & $53(24,1)$ & & $42(16,5-83)$ & $220(77,5)$ \\
\hline Beginn mit Teilpensum & $3(7,1)$ & $6(14,3)$ & $20(47,6)$ & $13(31,0)$ & & $45(34-103)$ & $42(14,8)$ \\
\hline Beginn mit Teilbelastung & $1(6,7)$ & $0(0,0)$ & $6(40,0)$ & $8(53,3)$ & & $84(42-92)$ & $15(5,3)$ \\
\hline fehlende Angaben & $1(14,3)$ & $1(14,3)$ & $1(14,3)$ & $4(57,1)$ & & $84(21-150)$ & $7(2,5)$ \\
\hline Invalidenrente (Voll/Teil) erhalten & & & & & 0,295 & & \\
\hline nein & $42(15,9)$ & $53(20,1)$ & $100(37,9)$ & $69(26,1)$ & & $44,5(20-84,5)$ & $264(93,0)$ \\
\hline ja & $0(0,0)$ & $1(33,3)$ & $0(0,0)$ & $2(66,7)$ & & $205(21-214)$ & $3(1,1)$ \\
\hline fehlende Angaben & $1(5,9)$ & $4(23,5)$ & $5(29,4)$ & $7(41,2)$ & & $56(27-91)$ & $17(6,0)$ \\
\hline Integritätsentschädigung erhalten & & & & & 0,221 & & \\
\hline nein & $39(18,1)$ & $45(20,8)$ & $79(36,6)$ & $53(24,5)$ & & $41,5(17,5-83)$ & $216(76,1)$ \\
\hline ja & $0(0,0)$ & $0(0,0)$ & $4(57,1)$ & $3(42,9)$ & & $69(61-192)$ & $7(2,5)$ \\
\hline fehlende Angaben & $4(6,6)$ & $13(21,3)$ & $22(36,1)$ & $22(36,1)$ & & $59(25-103)$ & $61(21,5)$ \\
\hline
\end{tabular}


Tab. 1 Fortsetzung.

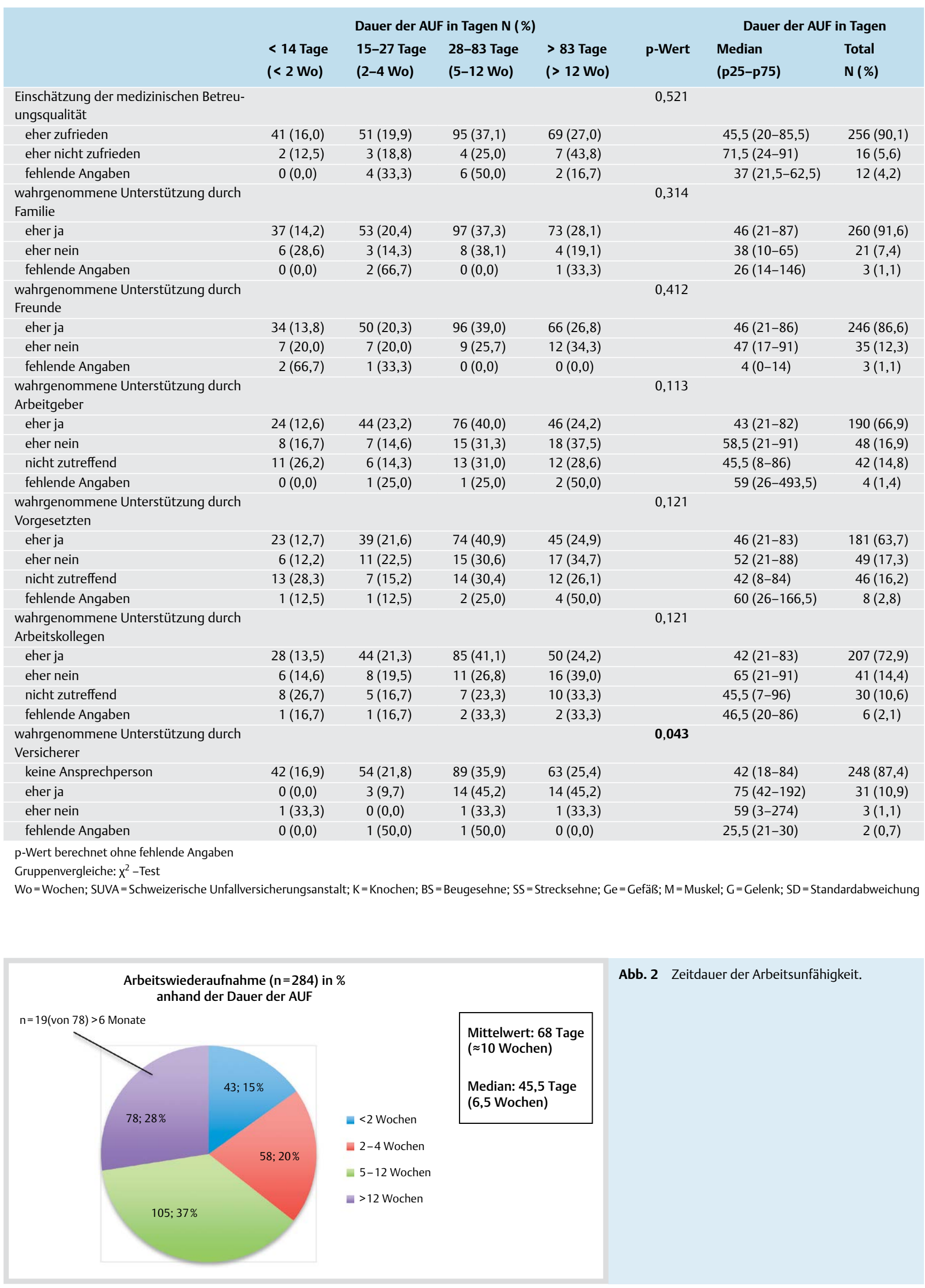


Zum Zeitpunkt der Befragung empfingen 2 der Studienteilnehmenden eine Teilrente, 7 haben aufgrund ihrer Handverletzung eine Integritätsentschädigung erhalten.

\section{Selbsteingeschätzte Funktion}

In $\odot$ Tab. $\mathbf{2}$ ist die selbsteingeschätzte Beurteilung der Funktion bezogen auf die Dauer der AUF dargestellt. Einen späteren Arbeitsbeginn zeigten Patienten, die zum Erhebungszeitpunkt mit ihrer Handfunktion, der Kraft und Geschicklichkeit eher unzufrieden waren, im QuickDASH Einschränkungen im Alltag angaben und sich aufgrund ihrer eingeschränkten Fähigkeiten als weniger nützlich einschätzten.

Die Werte des QuickDASH sind in $\odot$ Tab. 3 ersichtlich. Je später der Arbeitsbeginn erfolgte, desto mehr Einschränkungen wur- den auch noch zum Erhebungszeitpunkt von den Teilnehmenden wahrgenommen. Der mediane Wert des QuickDASH beträgt 4,5 (0-18,2), der Mittelwert 11,4 (SD 14,5).

\section{Multivariable Regressionsanalyse der Zeitdauer der AUF \\ $\nabla$}

Eine signifikante Einflussnahme auf die Zeitdauer der AUF hatten (auch nach der Adjustierung für mögliche und bekannte Störfaktoren) die Höhe des Verletzungsniveaus, die Anzahl der verletzten Regionen und der durchgeführten Sekundäreingriffe. Das Alter und die berufliche Tätigkeit blieben ebenfalls signifikant mit dem Outcome assoziiert. Hingegen war der Bildungs-

Tab. 2 Selbsteingeschätzte Funktion bezogen auf die Dauer der Arbeitsunfähigkeit (AUF).

\begin{tabular}{|c|c|c|c|c|c|c|c|c|c|}
\hline & \multicolumn{3}{|c|}{ Dauer der AUF in Tagen N (\%) } & \multirow[b]{2}{*}{$\begin{array}{l}>83 \text { Tage } \\
(>12 \text { Wo })\end{array}$} & \multirow[b]{2}{*}{ p-Wert } & \multicolumn{2}{|c|}{ Dauer der AUF in Tagen } & \multicolumn{2}{|c|}{$\begin{array}{l}\text { keine Angaben } \\
\text { Dauer der AUF }\end{array}$} \\
\hline & $\begin{array}{l}<14 \text { Tage } \\
\text { (< } 2 \text { Wo })\end{array}$ & $\begin{array}{l}\text { 15-27 Tage } \\
\text { (2-4 Wo) }\end{array}$ & $\begin{array}{l}\text { 28-83 Tage } \\
\text { (5-12 Wo) }\end{array}$ & & & $\begin{array}{l}\text { Median } \\
\text { (p25-p75) }\end{array}$ & $\begin{array}{l}\text { Total } \\
\text { N (\%) }\end{array}$ & & $\begin{array}{l}\text { Total } \\
\text { N (\%) }\end{array}$ \\
\hline $\begin{array}{l}\text { Zufriedenheit mit der } \\
\text { Funktion }\end{array}$ & & & & & 0,047 & & $n=284$ & $n=6$ & $n=290$ \\
\hline eher ja & $40(16,4)$ & $52(21,3)$ & $92(37,7)$ & $60(24,6)$ & & $42(18,5-83)$ & $244(85,9)$ & 4 & $248(85,5)$ \\
\hline eher nein & $3(7,5)$ & $6(15,0)$ & $13(32,5)$ & $18(45,0)$ & & $72,5(29,5-159)$ & $40(14,1)$ & 2 & $42(14,5)$ \\
\hline Zufriedenheit mit der Kraft & & & & & $<0,001$ & & $n=284$ & $n=6$ & $n=290$ \\
\hline eher ja & $43(17,6)$ & $54(22,1)$ & $92(37,7)$ & $55(22,5)$ & & $41,5(16,5-78)$ & $244(85,9)$ & 2 & $246(84,8)$ \\
\hline eher nein & $0(0,0)$ & $4(10,0)$ & $13(32,5)$ & $23(57,5)$ & & $91(50,5-185)$ & $40(14,1)$ & 4 & $44(15,2)$ \\
\hline $\begin{array}{l}\text { Zufriedenheit mit der } \\
\text { Beweglichkeit }\end{array}$ & & & & & 0,001 & & $n=284$ & $n=6$ & $n=290$ \\
\hline eher ja & $40(17,2)$ & $50(21,6)$ & $90(38,8)$ & $52(22,4)$ & & $41(17,5-78)$ & $232(81,7)$ & 4 & $236(81,4)$ \\
\hline eher nein & $3(5,8)$ & $8(15,4)$ & $15(28,9)$ & $26(50,0)$ & & $83,5(35-125)$ & $52(18,3)$ & 2 & $54(18,6)$ \\
\hline $\begin{array}{l}\text { Zufriedenheit mit dem } \\
\text { Aussehen }\end{array}$ & & & & & 0,155 & & $n=284$ & $n=6$ & $n=290$ \\
\hline eher ja & $40(17,0)$ & $47(20,0)$ & $88(37,5)$ & $60(25,5)$ & & $42(20-84)$ & $235(82,8)$ & 6 & $241(83,1)$ \\
\hline eher nein & $3(6,1)$ & $11(22,5)$ & $17(34,7)$ & $18(36,7)$ & & $60(22-146)$ & $49(17,3)$ & 0 & $49(16,9)$ \\
\hline QuickDASH & & & & & $<0,001$ & & $\mathrm{n}=282$ & $n=6$ & $\mathrm{n}=288$ \\
\hline 0-4\% (keine Einschränkung) & $28(22,4)$ & $29(23,2)$ & $47(37,6)$ & $21(16,8)$ & & $30(14-66)$ & $125(44,3)$ & 2 & $127(44,1)$ \\
\hline $\begin{array}{l}\text { 5-24\% (geringe Einschrän- } \\
\text { kung) }\end{array}$ & $13(12,4)$ & $20(19,1)$ & $43(41,6)$ & $29(27,6)$ & & $52(21-86)$ & $105(37,2)$ & 1 & $106(36,8)$ \\
\hline $\begin{array}{l}\text { 25-49\% (mittlere Ein- } \\
\text { schränkung) }\end{array}$ & $2(4,2)$ & $9(18,8)$ & $13(27,1)$ & $24(50,0)$ & & $83,5(29,5-151)$ & $48(17,0)$ & 1 & $49(17,0)$ \\
\hline $\begin{array}{l}\text { 50-95\% (hohe Einschrän- } \\
\text { kung) }\end{array}$ & $0(0,0)$ & $0(0,0)$ & $1(25,0)$ & $3(75,0)$ & & $157,5(82-187,5)$ & $4(1,4)$ & 2 & $6(2,1)$ \\
\hline $\begin{array}{l}\text { Wahrnehmung der eigenen } \\
\text { Nützlichkeit }\end{array}$ & & & & & $<0,001^{*}$ & & $n=284$ & $n=6$ & $n=290$ \\
\hline eher nicht eingeschränkt & $42(16,9)$ & $55(22,1)$ & $96(38,6)$ & $56(22,5)$ & & $41(18-80)$ & $249(87,7)$ & 3 & $252(86,9)$ \\
\hline eher eingeschränkt & $0(0,0)$ & $1(3,7)$ & $7(25,9)$ & $19(70,4)$ & & $104(69-192)$ & $27(9,5)$ & 3 & $30(10,3)$ \\
\hline fehlende Angaben & $1(2,3)$ & $2(25,0)$ & $2(25,0)$ & $3(37,5)$ & & $43(22-92)$ & $8(2,8)$ & 0 & $8(2,8)$ \\
\hline
\end{tabular}

Gruppenvergleiche: $\mathrm{x}^{2}$-Test; ${ }^{*} \mathrm{p}$-Wert berechnet ohne fehlende Angaben

$W_{0}=$ Wochen; $S \mathrm{D}=$ Standardabweichung

Tab. 3 QuickDASH bezogen auf die Dauer der Arbeitsabwesenheit in Tagen.

\begin{tabular}{|c|c|c|c|c|c|c|c|c|}
\hline & $\begin{array}{l}<14 \text { Tage } \\
\text { (<2 Wo) }\end{array}$ & $\begin{array}{l}\text { 15-27 Tage } \\
\text { (2-4 Wo) }\end{array}$ & $\begin{array}{l}\text { 28-83 Tage } \\
\text { (5-12 Wo) }\end{array}$ & $\begin{array}{l}>83 \text { Tage } \\
\text { (>12 Wo) }\end{array}$ & p-Wert & $\begin{array}{l}\text { Median (range) } \\
\text { QuickDASH } \\
\text { gesamt } \mathbf{n}=\mathbf{2 8 2}\end{array}$ & $\begin{array}{l}\text { Mittelwert (SD) } \\
\text { QuickDASH } \\
\text { gesamt } \mathbf{n}=\mathbf{2 8 2}\end{array}$ & $\begin{array}{l}\text { QuickDASH fehlenden } \\
\text { Angaben zur Zeitdauer } \\
\text { der Arbeitsunfähigkeit }\end{array}$ \\
\hline $\begin{array}{l}\text { QuickDASH } \\
\mathrm{n}=282\end{array}$ & $\mathrm{n}=43$ & $\mathrm{n}=58$ & $n=104$ & $n=77$ & $<0,001$ & & & $\mathrm{n}=6$ \\
\hline $\begin{array}{l}\text { Median } \\
\quad(p 25-p 75)\end{array}$ & $\begin{array}{l}2,3 \\
(0-9,1)\end{array}$ & $\begin{array}{l}3,6 \\
(0-15,9)\end{array}$ & $\begin{array}{l}4,5 \\
(0-11,9)\end{array}$ & $\begin{array}{l}11,4 \\
(2,3-29,5)\end{array}$ & & $4,5(0-18,2)$ & $11,4(14,5)$ & $\begin{array}{l}22,7 \\
(0-67)\end{array}$ \\
\hline Mittelwert (SD) & $5,1(7,3)$ & $9,8(12,5)$ & $8,9(12,1)$ & $17,6(16,5)$ & & & & $31,2(32,6)$ \\
\hline
\end{tabular}

p-Wert berechnet mit Kruskal-Wallis-Test

Wo $=$ Wochen; $S D=$ Standardabweichung 
grad nach dem Einfügen tätigkeits- und unterstützungsbezogener Merkmale nicht mehr signifikant mit der Zielvariablen verknüpft ( $\odot$ Tab. 4).

Merkmale wie Geschlecht, Verletzungstyp, Komplikationen, Herkunft, Versicherer, Art der verletzten Struktur, Verletzungsort, Erwerbsstatus, Anforderung an die Handfunktion, Unterstützung im privaten und im Arbeitsbereich waren im Regressionsmodell nicht signifikant mit der Dauer der AUF assoziiert. Die erklärte Varianz $\left(R^{2}\right)$ verbesserte sich durch Aufnahme der persönlichen und tätigkeitsbezogenen Merkmale in das Modell signifikant von 25,0\% im Modell 1 auf 39,1\% (Modell 2) bzw. auf 43,9\% (Modell 3). Die im Modell 4 mitberücksichtigten Unterstützungsfaktoren brachten kaum eine Erhöhung der Erklärbarkeit der Varianz (45,8\%).

\section{Diskussion}

$\nabla$

Hauptziel unserer Studie war es, die Zeitdauer der AUF und die Rückkehrrate Handverletzter in den Arbeitsprozess anhand klinischer und nicht-klinischer Faktoren zu untersuchen.

Um die Schwere einer Handverletzung zu erfassen wird in der Literatur vor allem ein Messinstrument (HISS: hand injury severity scoring system) zitiert, das eine grobe Einschätzung der voraussichtlichen Arbeitsunfähigkeit oder deren Dauer erlaubt [15-18]. Dieses Bewertungssystem hat einige Einschränkungen, so werden Gefäßläsionen nicht erfaßt und die Bewertung von Verletzungen der Haut im Vergleich zu motorischen und skelettalen Komponenten wird zu hoch gewichtet [19]. Zudem wird mit diesem Score nur die Verletzungsschwere des Ausgangsbefundes erfaßt, und nicht unterschieden zwischen einer Amputationsverletzung mit durchgeführter Replantation oder einer Amputationsverletzung mit Stumpfbildung. Die mittlere Dauer der Arbeitsunfähigkeit variiert hier massiv [20]. Auch nachträglich aufgetretene Komplikationen, die einen wichtigen Grund für die längere Arbeitsunfähigkeit darstellen, werden in diesem Score nicht abgebildet.

Um einen Vergleich unterschiedlicher Läsionen zu ermöglichen, wurden in unserer Studie der Verletzungstyp, die verletzte Struktur, das Verletzungsniveau (Endglied, Finger, Mittelhand, Handgelenk und 2/3 des Unterarms) und die Anzahl betroffener Regionen in den Berechnungen berücksichtigt ( $\bullet$ Tab. 1,4). $78,2 \%$ der Teilnehmenden weisen eine kombinierte Verletzungen und/oder Verletzungen der Nerven auf.

\section{Rückkehrrate und Zeitdauer der AUF}

In unserer klinikbasierten Studienpopulation von offenen Handverletzungen konnten wir eine hohe Rückkehrrate in den Arbeitsprozess aufzeigen. Über $72 \%$ der Handverletzten konnten innerhalb von 12 Wochen ihre Tätigkeit wieder aufnehmen, 91,7\% kehrten an ihren bisherigen Arbeitsplatz zurück.

Etliche Studien haben die Dauer der AUF hinsichtlich verschiedener Arten von Handverletzungen untersucht $[6,10,18,20]$. Skov ermittelte bei 802 Patienten mit Arbeitsunfällen, die sich innerhalb von 6 Monaten an einem Universitätsspital vorstellten, eine durchschnittliche Arbeitsabwesenheit von 6,1 (1-180) Tagen, was auf einen hohen Anteil an geringfügigen Verletzungen in der Primärversorgung hinweist [10].

Mit Verletzungen im Bereich des Handgelenks bzw. der Hand mit Beteilung von Nervenstrukturen befasste sich Bruyns. 48 von 81 Betroffenen nahmen innerhalb eines Jahres nach der Verletzung ihre Arbeit wieder auf, die durchschnittliche Dauer der
AUF betrug 219 Tage [6]. Es zeigte sich, dass diese Art der Verletzungen eine längere Heilungsdauer benötigten und zu Funktionsbeeinträchtigungen führen konnten, sodass sich die Wiederaufnahme der Arbeit verzögerte.

Wong [18], die in ihrer Studie 109 handtherapeutisch betreute Patienten mit unterschiedlichen Verletzungen einschloss, ermittelte eine durchschnittliche Zeitdauer der AUF von 52,5 Tagen und eine Rückkehrrate von 87,9\%. Ein Vergleich mit unseren Ergebnissen gestaltet sich schwierig, da Wong Nervenverletzungen nicht ausweist. Wachter et al. [20] ermittelten in ihrem Kollektiv von 184 Handverletzten (nur Arbeitsunfälle und Verletzungen distal des Karpus) eine mittlere AUF von 112 Tagen.

In der systematischen Review von Shi et al. [21], die 8 Studien einschloss, betrugen die durchschnittlichen Rückkehrraten nach 6 Monaten 57-98\%, in unserer Studie waren es nach diesem Zeitraum $93 \%$.

\section{Beeinflussende Faktoren}

Patienten mit distalen Handverletzungen, mit Verletzungen in weniger als 2 Regionen, ohne Sekundäreingriffe, über 55-jährig und ohne vorwiegend manuelle Tätigkeit wiesen eine kürzere Zeitdauer der AUF auf.

Dies zeigt in Übereinstimmung mit anderen Autoren, dass verletzungsrelevante Merkmale $[5,10,18,21]$ und die Anzahl an Nachfolgeoperationen [18] die Zeitdauer der AUF maßgeblich beeinflussten. Ebenso benötigten Verletzte, die manuelle Berufe ausüben, mehr Zeit für die Rückkehr in den Arbeitsprozess $[6,9,10]$.

Über den Einfluss des Alters auf die Dauer der AUF sind die Ergebnisse anderer Studien uneinheitlich. So gibt es Hinweise, dass jüngere Menschen früher an die Arbeit zurückkehren $[9,22]$, aber auch Untersuchungen, die unsere Resultate stützen $[11,23]$. Ein anderes Arbeitsethos, Erfahrungswissen, welches die Übernahme anderer Aufgaben erleichtert und die Angst vor einem Arbeitsplatzverlust können als mögliche Gründe für eine frühere Rückkehr älterer Menschen an den Arbeitsplatz gelten. Die Bildung hatte im Regressionsmodell nach dem Einfügen tätigkeitskeitsbezogener Merkmale keinen signifikanten Einfluss auf die Dauer der AUF gezeigt, dies wird auch in der systematischen Review von Shi et al. berichtet [21]. Es ist anzunehmen, dass Menschen mit einer höheren Bildung Arbeiten verrrichten, die manuell weniger fordernd sind.

Im Gegensatz zu MacKenzie's Studienergebnissen [9] hatte in unserer Analyse die bei einem hohen Prozentsatz der Teilnehmenden wahrgenommene soziale Unterstützung, keinen Einfluss auf die Zeitdauer der AUF. Der fehlende Bedarf an zusätzlicher sozialer Unterstützung könnte auf die kurze Arbeitsplatzabwesenheit von weniger als 6 Wochen bei ca. $50 \%$ der Studienteilnehmenden zurückzuführen sein. Dass soziale Unterstützung vor allem bei längerfristigen Beeinträchtigungen und kaum bei wenig invasiven chirurgischen Eingriffen eine wichtige Rolle spielt, wird in einer Literaturstudie belegt [24]. Neben den bearbeiteten Fragestellungen fällt auf, dass in unserer Studie 9 von 19 Handverletzten mit einer AUF von mehr als 6 Monaten von keiner für sie zuständigen Ansprechperson beim Versicherer wussten. Da die Kommunikation zwischen medizinischen Leistungserbringern, Patient und Arbeitsplatz als weiterer wichtiger Faktor im Prozess der Arbeitswiederaufnahme gilt [25], lässt sich hier Verbesserungspotenzial ausmachen.

Rüegg [12] analysierte 304 Datensätze von SUVA-Versicherten, die eine distale Radiusfraktur erlitten hatten, und konnte in ihrem Regressionsmodell statistisch signifikante Zusammen- 


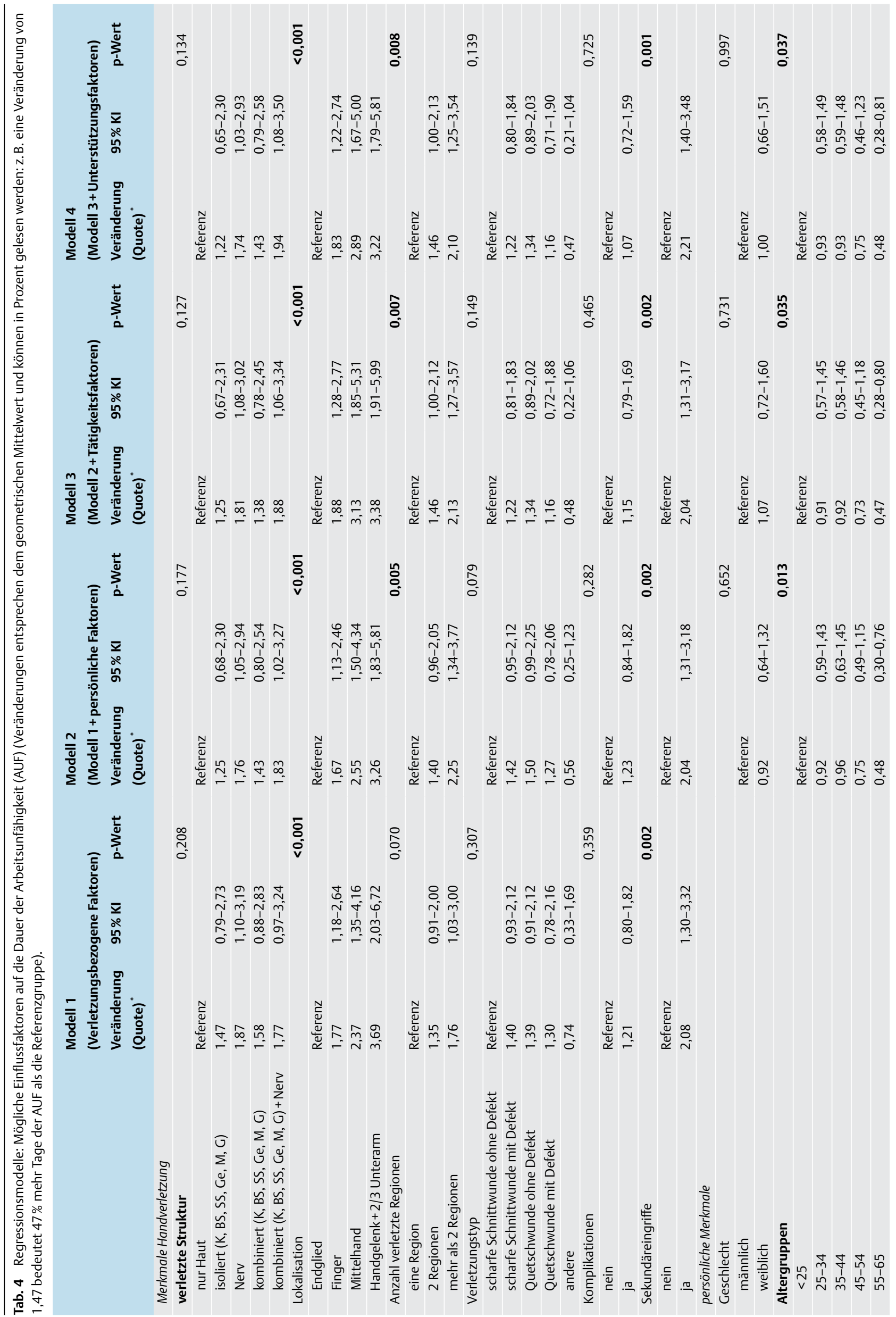






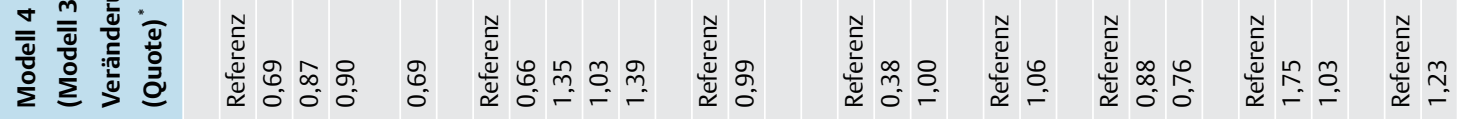



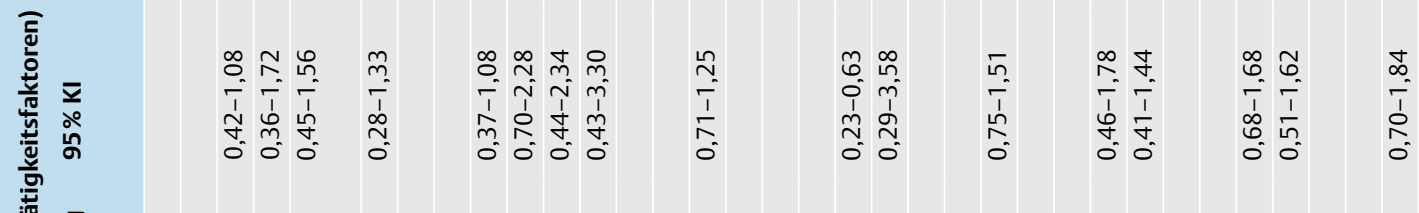
$m \stackrel{+}{\stackrel{ \pm}{*}}$

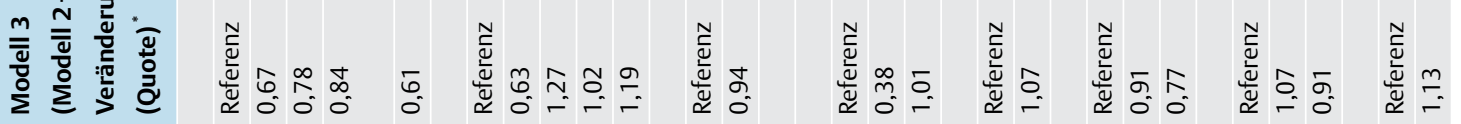

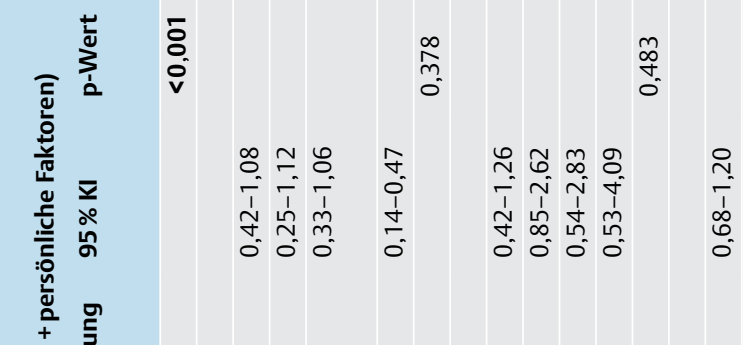

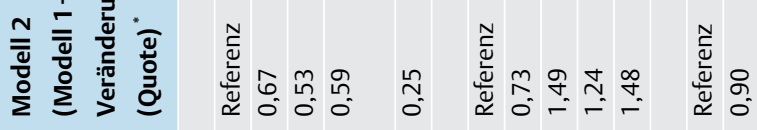
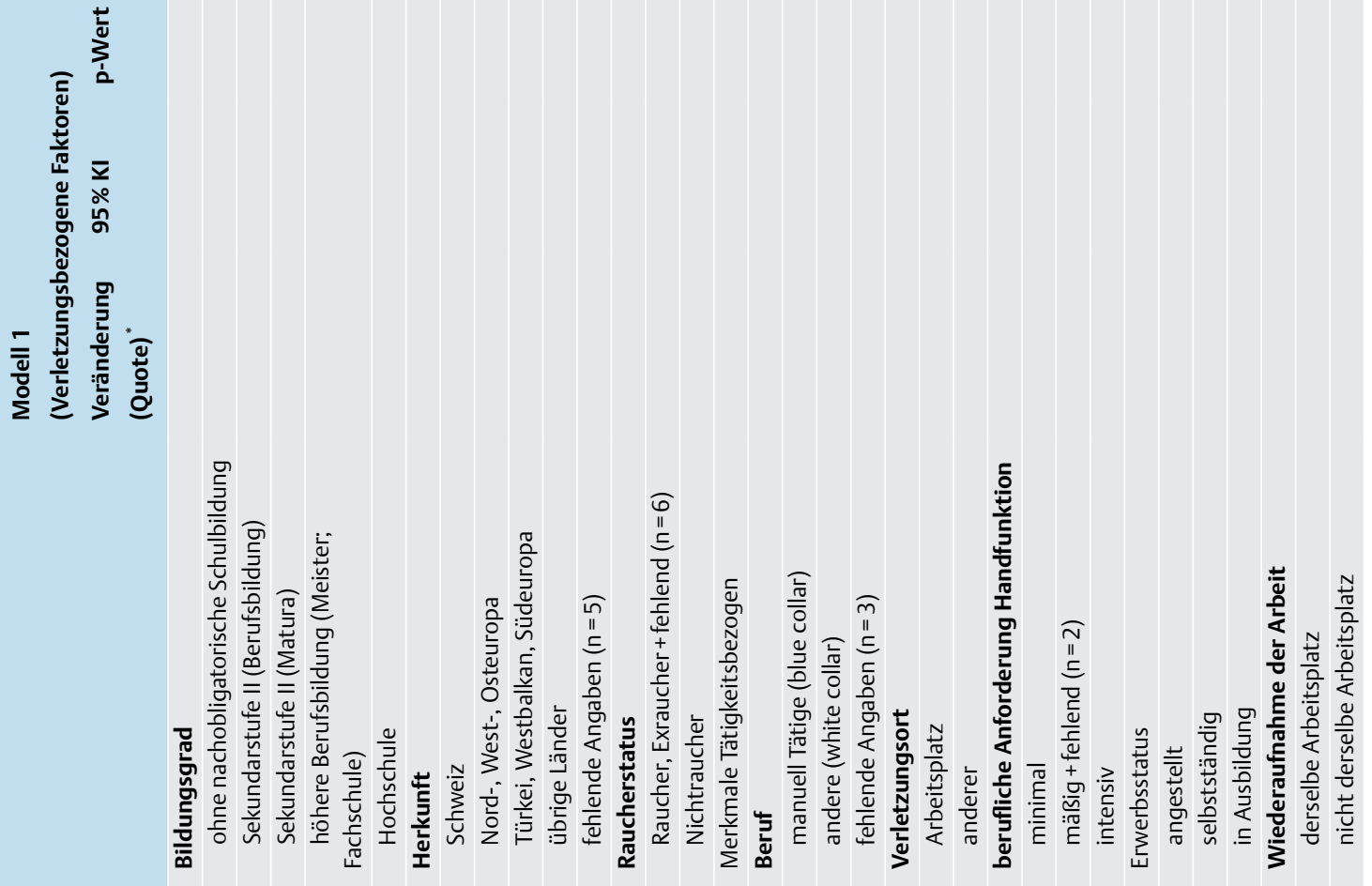





hänge unter anderem zwischen Nationalität und der Dauer der AUF nachweisen. In unserem Regressionsmodell waren keine signifikanten Verknüpfungen zwischen der Herkunft und der Dauer der AUF feststellbar. Durch die in unserem Modell mitberücksichtigte Schulbildung wurde dieser mögliche Störfaktoren kontrolliert. Der Ausländeranteil war aber mit 19\% wesentlich geringer als bei Rüegg (27\%).

Entgegen der üblichen Annahme, dass Selbstständigerwerbende aufgrund geringerer Versicherungsleistungen früher in den Arbeitsprozess zurückkehren [16], weisen unsere Ergebnisse für diese Erwerbsgruppe eine geringfügig längere AUF aus. Nicht erhoben wurden Angebote individuell angepasster Versicherungsmodalitäten für Selbstständigerwerbende als mögliche Einflussfaktoren.

Der niedrige mediane Wert des QuickDASH von 4,5 zeigt für die gesamte Studienpopulation eine gute Erholung von ihren Handverletzungen auf. Ursache für diesen sehr niedrigen Wert kann die im Sample eingeschlossene junge Population mit einem Durchschnittsalter von 38,9 Jahren sein.

Die große Variabilität in der vergleichbaren Literatur kann damit erklärt werden, dass sich die Studienpopulation, die Definition der Wiederaufnahme der Arbeit, die Studiendesigns und die Datenanalyse unterscheiden. Die meisten Studien wurden anhand homogener Studienpopulationen durchgeführt, wie Verletzungen am Arbeitsplatz, der Nerven, des Karpus oder des distalen Radius $[6,12,20]$. Dazu kommt, dass etliche Studien nur Determinanten von ein oder 2 möglichen beeinflussenden Kategorien untersuchten $[5,6,20,26]$. Unterschiede in den Gesundheitsund Versicherungssystemen verschiedener Länder und den gesetzlichen Bestimmungen, welche die Arbeitswiederaufnahme regeln, können weitere Gründe für die Differenzen in den Ergebnissen sein.

\section{Schwächen und Stärken}

Dies ist die erste in der Schweiz an einem Tertiärzentrum (Klinik mit Maximalversorgung) durchgeführte quantitative Studie, die die Rückkehr von Handverletzten an den Arbeitsplatz untersucht. Ein großes Patientenkollektiv und die hohe Antwortquote der Teilnehmenden können als Stärke gewertet werden. Als retrospektive Studie konzipiert zeigen unsere Ergebnisse lediglich einen Trend auf. Unser Patientenkollektiv, das auch Selbstständigerwerbende mit einschloss, beruht auf klinikbasierten Daten. Komplikationen und Sekundäreingriffe wurden ebenfalls erfasst.

Die Erhebung möglicherweise beeinflussender Faktoren zur Wiederaufnahme der Arbeit aus Patientensicht ist neu. Die bisher in der Schweiz durchgeführte Studie basierte auf Versicherungsdaten. Da die Erhebung nicht klinischer Faktoren mittels Fragbogen zu einem späteren Zeitpunkt erfolgte, ist jedoch ein recall-bias (Erinnerungsverzerrung) nicht auszuschließen. Mit 14 Monaten als kürzestem erfassten Beobachtungszeitraum kann allerdings davon ausgegangen werden, dass auch Menschen mit Verletzungen, die eine längere Heilungszeit erforderten, ihre Arbeit wieder aufgenommen haben.

Demografische Unterschiede ergeben sich zwischen Antwortenden und Nicht-Antwortenden. So sind Personen mit Schweizer Nationalität und ältere Menschen unter den Studienteilnehmenden häufiger vertreten.

Eine Kontrolle des Modus der handtherapeutischen Nachbehandlung (im Haus oder extern), die ebenfalls eine wichtige Rolle für das funktionelle Ergebnis spielt, war aufgrund des retrospektiven Designs nicht möglich.
Wegen begrenzter Ressourcen konnten wir lediglich Patienten berücksichtigen, welche über ausreichende Deutschkenntnisse verfügten. Die Ergebnisse der Studie können daher nicht auf alle Bevölkerungteile übertragen werden. Unterschiedliche Versicherungssysteme und gesetzliche Bestimmungen, die die Arbeitswiederaufnahme regeln, und ein anderer Arbeitsethos können in anderen Ländern zu anderen Ergebnissen führen.

\section{Schlussfolgerung \\ $\nabla$}

Verletzungsmerkmale und Alter erwiesen sich in unserer Studie als 2 wichtige Einflussfaktoren auf die Dauer der AUF. Da beide nicht modifizierbar sind, wird die primäre fachgerechte Rekonstruktion der verletzten Strukturen zum einem wichtigen Garanten für ein gutes funktionelles Ergebnis. Da sich die Natur der erfolgten Verletzung als gegeben erweist, müssen unsere Anstrengungen einerseits der Prävention und andererseits einer sachgemäßen frühen klinischen und therapeutischen Rehabilitation gelten.

Als dritter Faktor spielte die ausgeübte Tätigkeit bei der Wiederaufnahme der Arbeit eine maßgebende Rolle. Arbeit, als eine der Hauptquellen persönlicher Befriedigung und sozialer Interaktion, kann bei einem temporären oder permanenten Verlust einen erheblichen Einfluss auf das Selbstbewusstsein Erwerbstätiger ausüben [27]. Dieser Umstand spricht nebst den volkswirtschaftlichen Folgekosten einer Handverletzung dafür, die AUF möglichst kurz zu halten. Handverletzungen verlangen zwar eine bestimmte Heilungsdauer, dennoch wäre zu prüfen, ob sich durch eine optimierte Kommunikation und Kooperation zwischen Patient, Arbeitsplatz, Versicherer und medizinischem Personal entscheidende Weichenstellungen in der Rehabilitation vornehmen ließen mit dem Ziel einer abgestuften und individuell angepassten früheren Wiederaufnahme der Arbeit. Eine frühzeitige Kombination medizinischer und beruflicher Maßnahmen unter Einbezug der Unterstützungssysteme stellt eine wesentliche Voraussetzung für eine effektive und erfolgreiche Rehablitation Handverletzter dar. Studien, die dies bei Handverletzten untersuchten, liegen bisher nicht vor und wären die Konsequenz.

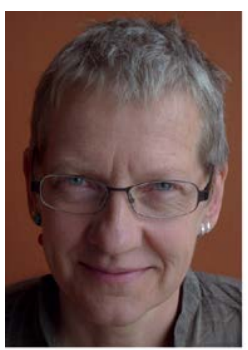

Elisabeth Oberfeld

1979 Diplom als Ergotherapeutin, Wien; 1980-2000 Tätigkeit als Ergotherapeutin in verschiedenen Fachbereichen mit Schwerpunkt Neurologie und Handchirurgie in der Schweiz; 2001 Lizentiat in Ethnologie an der Universität Bern; seit 2003 in der Handtherapie der Universitätsklinik für Plastische- und Handchirurgie, Inselspital Bern; 2012 Master of Public Health (MPH), Universitäten Basel, Bern, Zürich; 2012 Übernahme der Funktion als Therapie-Expertin.

Interessenkonflikt: Es besteht kein Interessenkonflikt, die Studie wurde im Rahmen des Abschlusses zum Master of Public Health durchgeführt. 


\section{Literatur}

1 Schweizerische Gesellschaft für Handchirurgie (SGH). http://www. swisshandsurgery.ch/patienteninfo.aspx accessed 01.09.2011

2 Siegrist S, Bisig B.. Unfallbedingte Verletzungen. In: Gutzwiller F, Paccaud F, Hrsg. Sozial- und Präventivmedizin Public Health. 3. Aufl. Bern: Verlag Hans Huber, 2007; 295-302

3 Koordinationsgruppe für die Statistik der Unfallversicherung UVG (KSUV), Hrsg. Unfallstatistik 2014. Luzern: Sammelstelle für die Statistik der Unfallversicherung UVG (SSUV), 2014

4 Bauer R. EU Injury Database (EU IDB) - Prevention oriented injury surveillance - example hand injuries. "Hand in Prevention" $1^{\text {st }}$ EUROPEAN HAND INJURY PREVENTION CONGRESS; 25-27 June 2009; Bursa, Turkey

5 Urso-Baiarda F, Lyons RA, Laing JH et al. A prospective evaluation of the Modified Hand Injury Severity Score in predicting return to work. Int J Surg 2008; 6: 45-50

6 Bruyns $C N$, Jaquet JB, Schreuders TA et al. Predictors for return to work in patients with median and ulnar nerve injuries. J Hand Surg Am 2003; 28: 28-34

7 Bear-Lehman J. Factors affecting return to work after hand injury. Am J Occup Ther 1983; 37: 189-194

8 Buechler $U$. Funktionelle Interaktionen im Heilungsablauf kombinierter Verletzungen der Hand: Klinische Untersuchungen am Beispiel des Fingergrundgliedes [Habilitation]. Bern: Universität Bern, 1988; 324

9 MacKenzie EJ, Morris JA Jr, Jurkovich GJ et al. Return to work following injury: the role of economic, social, and job-related factors. Am J Public Health 1998; 88: 1630-1637

10 Skov O, Jeune B, Lauritsen JM et al. Time off work after occupational hand injuries. J Hand Surg Br 1999; 24: 187-189

11 Pransky GS, Benjamin KL, Savageau JA et al. Outcomes in work-related injuries: a comparison of older and younger workers. Am J Ind Med 2005; 47: 104-112

12 Rüegg D. The Management of Distal Radius Fractures in the Swiss Working Population: Epidemiology and Costs: Thesis for the Degree of Master of Public Health, Interuniversitäres Weiterbildungsprogramm Public Health (MPH). Universitäten Basel, Bern, Zürich: 2004

13 de Putter CE, Selles RW, Polinder $S$ et al. Economic impact of hand and wrist injuries: health-care costs and productivity costs in a population-based study. J Bone Joint Surg Am 2012; 94: e56

14 Scholz-Odermatt S. Sammelstelle für Statistik der Unfallversicherungen (UVG), Luzern. Persönliche Kommunikation 2. 9. 2013
15 Campbell DA, Kay SP. The Hand Injury Severity Scoring System. J Hand Surg Br 1996; 21: 295-298

16 Opsteegh L, Reinders-Messelink HA, Schollier D et al. Determinants of return to work in patients with hand disorders and hand injuries. J Occup Rehabil 2009; 19: 245-255

17 Matsuzaki $\mathrm{H}$, Narisawa $\mathrm{H}$, Miwa $\mathrm{H}$ et al. Predicting functional recovery and return to work after mutilating hand injuries: usefulness of Campbell's Hand Injury Severity Score. J Hand Surg Am 2009; 34: 880-885

18 Wong JY. Time off work in hand injury patients. J Hand Surg Am 2008; 33: 718-725

19 Saxena P, Cutler L, Feldberg L. Assessment of the severity of hand injuries using "hand injury severity score", and its correlation with the functional outcome. Injury 2004; 35: 511-516

20 Wachter NJ, Gulke J, Krischak GD et al. Einschätzung der Verletzungsschwere sowie der Dauer der Arbeitsunfähigkeit bei Handverletzungen mit dem HISS-Bewertungsschema. Handchir Mikrochir Plast Chir 2005; 37: 238-244

21 Shi $Q$, Sinden $K$, MacDermid JC et al. A systematic review of prognostic factors for return to work following work-related traumatic hand injury. J Hand Ther 2014; 27: 55-62

22 Hwang YF, Chen-Sea MJ, Chen CL. Factors related to return to work and job modification after a hand burn. J Burn Care Res 2009; 30: 661-667

23 Hou WH, Tsauo JY, Lin CH et al. Worker's compensation and returnto-work following orthopaedic injury to extremities. J Rehabil Med 2008; 40: 440-445

24 Rosenberger PH, Jokl P, Ickovics J. Psychosocial factors and surgical outcomes: an evidence-based literature review. J Am Acad Orthop Surg 2006; 14: 397-405

25 Kosny A, Franche RL, Pole J et al. Early healthcare provider communication with patients and their workplace following a lost-time claim for an occupational musculoskeletal injury. J Occup Rehabil 2006; 16 : 27-39

26 Rusch MD, Dzwierzynski WW, Sanger JR et al. Return to work outcomes after work-related hand trauma: the role of causal attributions. J Hand Surg Am 2003; 28: 673-677

27 Grob M, Papadopulos NA, Zimmermann A et al. The psychological impact of severe hand injury. J Hand Surg Eur Vol 2008; 33: 358-362 